\title{
6D magnetic field distribution measurements of permanent magnets with magnetic field camera scanner
}

\author{
Dr. K. Vervaeke \\ Magcam NV, Romeinse straat 18, B-3001 Leuven, Belgium, \\ koen.vervaeke@magcam.com
}

\begin{abstract}
:
We present state-of-the-art high resolution 3D magnetic field mapping on permanent magnets of the (Bx, By, Bz) magnetic field vector components in 3D space, resulting in measurement data with 6 degrees of freedom. These measurements are performed using a high speed 3D magnetic field camera, featuring an integrated 2D Hall sensor array, mounted on a 3-axis mechanical scan stage with a scan range of $300 \times 300 \times 300 \mathrm{~mm}^{3}$ with which measurement speeds of $120 \mathrm{~mm}^{2} / \mathrm{s}$ are obtained with a spatial resolution of $0.1 \mathrm{~mm} \times 0.1 \mathrm{~mm}$. Volume $3 \mathrm{D}$ magnetic field distribution measurements of sensor magnets are performed and analyzed using powerful and versatile data analysis software, which allows virtually unlimited possibilities for the data analysis and quality control of permanent magnets, both in R\&D, incoming magnet inspection and production.
\end{abstract}

Key words: magnet inspection, magnetic field camera, Hall sensor 2D array, magnetic field mapping, sensor magnet.

\section{Introduction}

Fast and accurate quality inspection of permanent magnets is increasingly important in development, production and quality control of ever more accurate and reliable sensors as widely used in automotive applications, more efficient electric drives for e.g. electric vehicles, new medical devices, consumer electronics and many other magnet applications. Furthermore, in many high-end applications a 100\% magnet quality control is desired, but not feasible with classical magnetic measurement equipment. Also in sensor R\&D there is a need for fast and advanced magnet characterization tools for developing next-generation sensor concepts and assemblies.

Recently interest has grown in the area of 3axis magnetic field distribution measurements [1-5]. Measuring the spatial distribution of the full vector magnetic field $(\mathrm{Bx}, \mathrm{By}, \mathrm{Bz})$ opens a new dimension of possibilities in the inspection of permanent magnets. From the Cartesian components, a range of derived magnetic field quantities are readily derived, such as $B$ (full field), Bxy (field in the $X Y$ plane), the azimuth angle (angle of the field vector in the XY plane) and polar angle (angle of the field vector out of the $X Y$ plane). These derived quantities are often exactly the important magnet characteristics that determine the performance of the magnet in the end application.
We show how a magnetic field camera based scan system can map the 3D field distribution in 3D space with micrometer spatial resolution over areas up to $300 \times 300 \times 300 \mathrm{~mm}^{3}$ with an unprecedented speed of $80 \mathrm{~mm}^{2} / \mathrm{s}$, which is about 30x faster than alternative methods.

The resulting data contains an immense amount of information about the magnet's quality. Much of this information is not readily visible in the data, but is extracted using advanced data analysis algorithms via powerful data analysis software [1,2,5-8]. The data analysis configuration can be fully customized, automated and saved for use in both R\&D and production inspection of permanent magnets and magnet assemblies of all types.

\section{Three-Axis Magnetic Field Camera}

Magcam developed the magnetic field camera technology for inspection of permanent magnets, based on a high resolution and high speed quantitative 2D mapping of the magnetic field distribution of a magnet, using a patented sensor chip with an integrated $2 \mathrm{D}$ array of microscopic Hall sensors [6-9]. A further development of this technology was a threeaxis magnetic field camera system ('MiniCube3D', see Fig. 1), which makes the three components of the magnetic field distribution available in a 2D area of $12.7 \mathrm{~mm} x$ $12.7 \mathrm{~mm}$, with $0.1 \mathrm{~mm}$ pixel resolution, equaling $128 \times 128=16384$ measurement points [1-3]. 


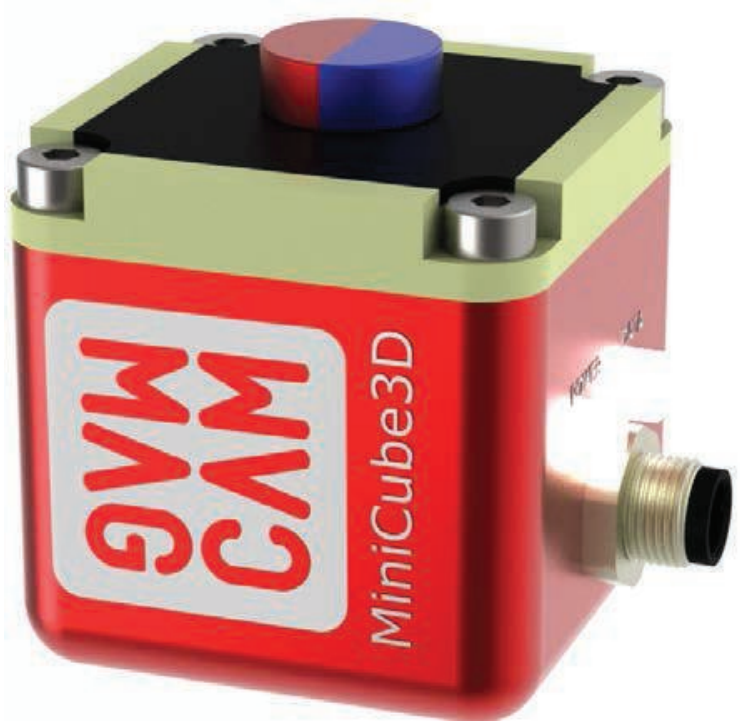

Fig. 1. Magcam MiniCube3D three-axis magnetic field camera with a two-pole axially magnetized cylindrical sensor magnet on top.

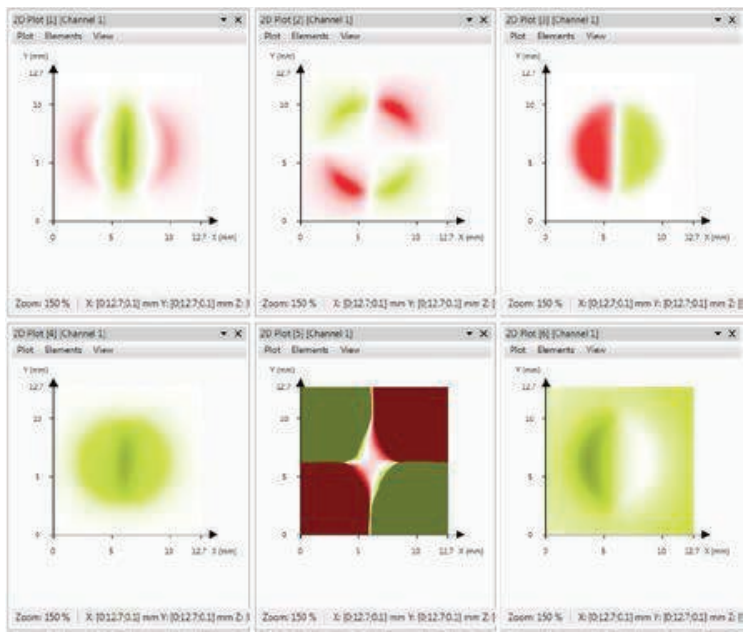

Fig. 2. Measured 3D magnetic field distribution of the magnet shown in Fig. 1. Measurement area: $12.7 \mathrm{~mm}$ $x \quad 12.7 \mathrm{~mm}$, spatial resolution: $0.1 \mathrm{~mm}$ in $X$ and $Y$. Components: Top row: Bx, By, Bz, Bottom row: $B$, azimuth angle, polar angle.

A full resolution frame is recorded in less than one second. It is important to mention that the pixels on one magnetic field camera module are read out in sequential order, whereby each pixel needs about 50 microseconds measurement time. This means that one can calculate the total needed time for recording one frame by simply multiplying the total number of pixels in the frame by the time per pixel of 50 microseconds. The measurement times for some special cases are listed in Tab. 1.
Tab. 1. Measurement times for various submatrices of the sensor matrix in one camera module

\begin{tabular}{|c|c|}
\hline Measured pixel matrix & time \\
\hline Full range, full resolution & $800 \mathrm{~ms}$ \\
\hline Full range, half resolution & $200 \mathrm{~ms}$ \\
\hline Single line, full resolution & $6.4 \mathrm{~ms}$ \\
\hline Single line, half resolution & $3.2 \mathrm{~ms}$ \\
\hline
\end{tabular}

\section{Mechanical XYZ scanning of a MiniCube3D magnetic field camera}

In order to map the 3D magnetic field distribution in a large area and/or a volume, the MiniCube3D magnetic field camera is mounted onto a $\mathrm{XYZ}$ scan stage and to sequentially measure multiple small-scale magnetic field maps which are subsequently stitched together to obtain a large area image.

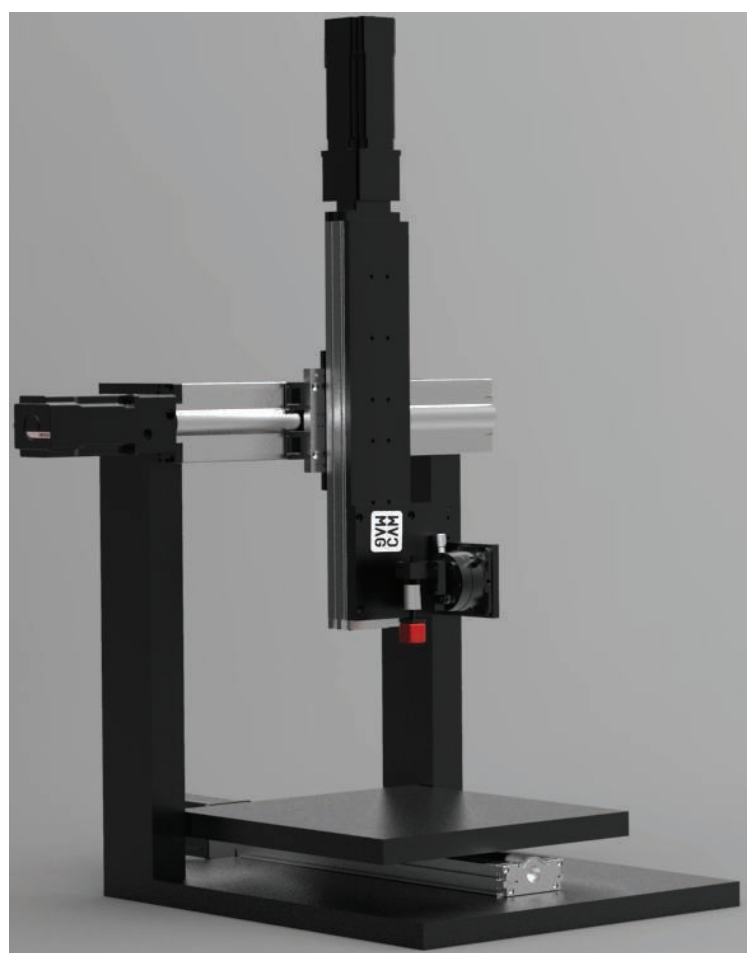

Fig. 3. Motorized $X Y Z$ scanner with integrated MiniCube $3 D$ magnetic field camera.

In order to accurately stitch the different measured images into a larger image, the scan step (in $X$ and $Y$ directions) is taken to be exactly equal to the corresponding size of the measurement area (i.e. $12.7 \mathrm{~mm}$ ). In this case there is exactly one pixel overlap between consecutive images. The quality of the resulting stitched image depends then on the accuracy of the scan stage and the alignment of the sensor $X$ and $Y$ axes to those of the scan stage. With a positioning accuracy in the micrometer range and correspondingly accurate orthogonality of 
the mechanical axes, this condition is fulfilled with the Magcam XYZ scanner.

Since the intrinsic measurement speed of a magnetic field camera (without moving parts) is relatively high (i.e. $12.7 \times 12.7 \mathrm{~mm}^{2} \quad / \quad 0.8$ seconds in full resolution) large areas can quickly be measured using this technique.

The total measurement time for an area $A$ is equal to

$T_{\text {total }}=\left(T_{\text {sin gle }}+T_{\text {scanstep }}\right) * A / A_{\text {sensor }}$

where

$T_{\text {total }}$ is the total measurement time,

$T_{\text {single }}$ is the time needed to measure a single $12.7 \mathrm{~mm} \times 12.7 \mathrm{~mm}$ image,

$A$ is the area to be measured,

$A_{\text {sensor }}$ is the area of the sensor, i.e. $12.7 \mathrm{~mm} \times 12.7 \mathrm{~mm}$.

Expression (1) shows that the total measurement time increases proportionally to the area to be measured. Based on this formula we can calculate quantitative measurement times for a few realistic cases.

Concerning the time per single measurement, we take the following approach. In practice, magnetic field camera measurements are often performed with half spatial resolution (i.e. $0.2 \mathrm{~mm}$ ) instead of full resolution (i.e. $0.1 \mathrm{~mm}$ ), especially for larger magnets. This means that only each second pixel is measured in $X$ - and $Y$-directions, resulting in only $1 / 4$ of all pixels being effectively read-out. This also means that the measurement time per frame is four times smaller, i.e. about $0.2 \mathrm{~s} /$ frame. On the other hand, multiple frames are usually recorded and averaged in order to reduce measurement noise. A realistic number of averages would be 5 , resulting in a total measurement time per frame of 1 second, which is comparable with the time for a single frame at full resolution.

The second parameter in the expression above is the time needed for a mechanical scan step, which is $12.7 \mathrm{~mm}$ in either the $X$ or $Y$ direction. The industrial axes used in the XYZ scanner allow high accelerations and speeds. Realistic speeds are in the range of $150 \mathrm{~mm} / \mathrm{s}$, making a step time of $0.1 \mathrm{~s}$ possible. The total time for a single measurement + scan step is thus about 1.1s. The total measurement time for a few realistic areas is calculated in Tab. 2.
Tab. 2. Total measurement times for different areas when mechanically scanning a single magnetic field camera on an $X Y$-stage. Results valid for $0.1 \mathrm{~mm}$ resolution with one measurement frame per step or $0.2 \mathrm{~mm}$ resolution with 5 averaging frames per step.

\begin{tabular}{|c|c|c|c|}
\hline Meas. area & \#steps & Time/step & Total time \\
\hline $12 \times 24 \mathrm{~mm}^{2}$ & 2 & $1.1 \mathrm{~s}$ & $2.2 \mathrm{~s}$ \\
\hline $24 \times 24 \mathrm{~mm}^{2}$ & 4 & $1.1 \mathrm{~s}$ & $4.4 \mathrm{~s}$ \\
\hline $24 \times 48 \mathrm{~mm}^{2}$ & 8 & $1.1 \mathrm{~s}$ & $8.8 \mathrm{~s}$ \\
\hline $48 \times 48 \mathrm{~mm}^{2}$ & 16 & $1.1 \mathrm{~s}$ & $17.6 \mathrm{~s}$ \\
\hline
\end{tabular}

These measurement speeds are about 30 times faster than those obtained with alternative methods, where one single (3D) Hall sensor is scanned over the same area with the same resolution. Advantages of the Magcam method are:

- 30x faster measurements compared to single sensor scanners

- Stable measurments (no mechanical vibrations) since the camera is stationary during each measurement step

- The ability to perform multiple averaging measurements at each position

Even higher scan speeds can be obtained when combining multiple MiniCube cameras on the scanner as described in [7].

The obtained measurement times of Tab. 1 are compatible with typical requirements of inline inspection stations. It becomes therefore a realistic option to perform such a $100 \%$ inline quality control of large magnets and magnetic assemblies.

\section{D volume measurements}

The combination of a three-axis magnetic field camera measuring the distribution of the $\mathrm{Bx}$, By, Bz components in a $2 \mathrm{D}$ plane, and a mechanical scanner able to scan in the $Z$ direction, allows measurements of sixdimensional magnetic field maps, i.e. the spatial distribution of the three magnetic field components in 3D space. An example of a typical application is a rotary encoder magnet, where the magnetic field distribution in $3 D$ space is relevant for finding the optimum position to place a magnetic sensor in a sensor system. A measured 6D Magcam magnetic field map is shown in Fig. 4. 


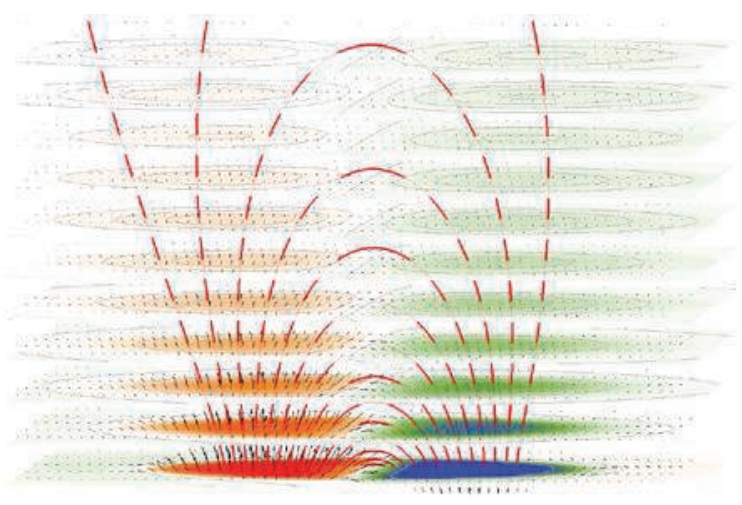

Fig. 4. 6D magnetic field distribution of a two-pole axially magnetized cylindrical sensor magnet $(8 \mathrm{~mm}$ diameter)

\section{Conclusion}

We presented state-of-the-art high resolution 3D magnetic field mapping on permanent magnets of the (Bx, By, Bz) magnetic field vector components in $3 \mathrm{D}$ space, resulting in measurement data with 6 degrees of freedom. These measurements are performed using a high speed 3D magnetic field camera, featuring an integrated 2D Hall sensor array, mounted on a 3-axis mechanical scan stage with a scan range of $300 \times 300 \times 300 \mathrm{~mm}^{3}$ with which measurement speeds of $120 \mathrm{~mm}^{2} / \mathrm{s}$ are obtained with a spatial resolution of $0.1 \mathrm{~mm} \times$ $0.1 \mathrm{~mm}$. Volume 3D magnetic field distribution measurements of sensor magnets are performed and analyzed using powerful and versatile data analysis software, which allows virtually unlimited possibilities for the data analysis and quality control of permanent magnets, both in R\&D, incoming magnet inspection and production.

These results show that the magnetic field camera technology is scalable to larger areas and volumes, whereby its inherent speed and spatial resolution are not compromised. These characteristics make the magnetic field camera technology reference for inspection of permanent magnets, applicable for a wide range of applications and suitable for automated inline inspection in production lines, quality control and R\&D.

\section{References}

[1] K. Vervaeke, "3-axis magnetic field camera for ultrafast and high resolution inspection of permanent magnets" Conference Proceedings of 4th International Electric Drives Production Conference (E|DPC 2014), 30 September - 1 October 2014, Nürnberg

[2] K Vervaeke, Conference Proceedings of 17. ITG / GMA-Fachtagung "Sensoren und Messsysteme“, 3.-4. Juni 2014, Nürnberg, P5.2

[3] www.magcam.com
[4] D R Popovic et al., IEEE Transactions on Instrumentation and Measurement, Vol. 56, no.4, August 2007

[5] http://www.iis.fraunhofer.de/en/pr/2013/20130610 _Fraunhofer-Preis.html

[6] K. Vervaeke, Conference Proceedings of 3rd International Electric Drives Production Conference (E|DPC 2013), 29-30 October 2013, Nürnberg, ISBN 978-1-4799-1102-8, pp. 38

[7] K. Vervaeke, Conference Proceedings of 16th International Conference on Sensors and Measurement Technology, 14-16 May 2013, Nürnberg (SENSOR 2013), DOI 10.5162/sensor2013/A7.1

[8] K. Vervaeke, Conference Proceedings of 2nd Inter-national Electric Drives Production Conference (E|DPC 2012), 15-18 October 2012, Nürnberg, ISBN 978-1-4673-3006-0, pp. 186

[9] K. Vervaeke, Conference Proceedings of 16. GMA/ITG Fachtagung Sensoren Und Messsysteme 2012, 22. und 23. Mai 2012, Nürnberg, 'Magnetic field camera for fast - high resolution inline magnet inspection', 2.3.1 